\title{
Atrial arrhythmias and pacing after orthotopic heart transplantation: bicaval versus standard atrial anastomosis
}

\author{
S C D Grant, M A Khan, E B Faragher, N Yonan, N H Brooks
}

\begin{abstract}
Background-Right and left atrial configuration is more normal when the donor left atrium is anastomosed to a recipient left atrial cuff with direct anastomoses of the donor and recipient vena cavas on the right side. The right atrium and sinus node may be less disturbed by the technique of bicaval anastomosis than by the standard procedure.
\end{abstract}

Objective-To compare the incidence of atrial arrhythmias and pacing after bicaval and standard anastomoses.

Methods-75 patients had heart transplants between January 1991 and December 1993. The notes were reviewed. Nine patients who died within the first 30 days were excluded from further analysis (seven patients with standard anastomoses, one with bicaval anastomosis, and one with a hybrid technique).

Results-66 patients survived for more than 30 days. Thirty five patients had standard anastomoses and 31 bicaval anastomoses. Atrial tachyarrhythmias (atrial fibrillation, atrial flutter, atrial tachycardia, or supraventricular tachycardia) occurred on four days in three patients in the bicaval group compared with 27 days in 13 patients in the standard group $(P=0.009)$. The relative risk of atrial tachyarrhythmias with standard anastomosis was $5.52(P=0.015)$ compared with that of bicaval anastomosis. Atrial tachyarrhythmias requiring treatment occurred less often in the bicaval group (four episodes in three patients in the bicaval group and eight episodes in four patients in the standard group), and fewer patients with a bicaval anastomosis required temporary pacing (pacing on 20 days in 10 patients in the bicaval group, but pacing on 49 days in 16 patients in the standard group) and late permanent pacing (no patients in the bicaval group and three patients in the standard group), although these differences were not statistically significant. Patients in the bicaval group were discharged from hospital sooner than those in the standard group (mean $24 \cdot 1 v 29 \cdot 1$ days, $P=0.024$ ). Conclusions-The technique of bicaval anastomosis, in addition to theoretical advantages from maintaining a more normal atrial configuration, has a lower incidence of postoperative atrial tachyarrhythmias, may reduce the need for pacing, and allows earlier discharge from hospital.

(Br Heart f 1995;74:149-153)

Keywords: bicaval atrial anastomosis; atrial arrhythmias; pacing; transplantation

The standard surgical technique for implantation of the transplanted heart described by Lower $e t a l^{1}$ involves excision of the recipient heart with division of the atrial septum, leaving cuffs of the right and left atrium and the great vessels to which the suitably trimmed donor heart is sutured. This technique is relatively simple and quick, and provides access to the anastomoses in cases of postoperative bleeding. It has the disadvantage that the atrial anatomy is distorted. Theoretical disadvantages of this distortion are that function of the tricuspid and mitral valves may be affected, as well as the atrial contribution to ventricular filling. Moreover the atrial surgery which involves substantial incision and suturing of the donor anterior right atrial wall has the potential to disturb the sinus node and produce an arrhythmogenic substrate. An alternative technique involving the use of a small left atrial cuff with anastomoses of the donor and recipient cavas has recently been described at this institution. ${ }^{2}$ We have compared our results using bicaval and standard anastomoses to determine the incidence of postoperative pacing and atrial arrhythmias.

Patients and methods

SURGICAL TECHNIQUE

The technique of bicaval anastomosis has been described in detail elsewhere. ${ }^{2}$ It was first introduced here in early 1991 . Since then bicaval and standard anastomoses have been used. Allocation to technique was on an alternate basis; there was a prospective intention to compare the two groups but formal randomisation was not initially undertaken. The principal contraindication to bicaval anastomosis was inadequate caval length available on the harvested donor organ.

\section{ELECTROCARDIOGRAM MONITORING}

Continuous telemetric monitoring was performed for a minimum of 7 days in all patients, and for longer periods in those in whom it was thought necessary. Thereafter 12 lead electrocardiograms were performed two to three times weekly until discharge. 
Table 1 Characteristics of standard and bicaval groups

\begin{tabular}{|c|c|c|c|}
\hline & Standard & Bicaval & $p$ Value \\
\hline $\begin{array}{l}\text { No of patients who died } \\
\text { No of patients }\end{array}$ & $75(16 \cdot 7 \%)$ & $2(6 \cdot 1 \%)$ & $0 \cdot 28$ \\
\hline $\begin{array}{l}\text { No or patients } \\
\text { Age at transplantation }\end{array}$ & $\begin{array}{l}35 \\
49 \cdot 2(45 \cdot 1 \text { to } 53 \cdot 4)\end{array}$ & $44 \cdot 1(39 \cdot 3$ to $49 \cdot 0)$ & $0 \cdot 106$ \\
\hline \multicolumn{4}{|l|}{ Sex } \\
\hline $\begin{array}{l}\text { males } \\
\text { females }\end{array}$ & $\begin{aligned} 31(88 \cdot 6 \%) \\
4(11 \cdot 4 \%)\end{aligned}$ & $\begin{array}{r}25(80 \cdot 6 \%) \\
6(19 \cdot 4 \%)\end{array}$ & $0 \cdot 158$ \\
\hline \multicolumn{3}{|l|}{ Diagnosis (patients) } & \multirow[t]{4}{*}{$0 \cdot 158$} \\
\hline Idiopathic dilated cardiomyopathy & $9(25 \cdot 7 \%)$ & $14(45 \cdot 2 \%)$ & \\
\hline Ischaemic heart disease & $24(68 \cdot 6 \%)$ & $14(45 \cdot 2 \%)$ & \\
\hline Other & $2(5 \cdot 7 \%)$ & $3(9 \cdot 7 \%)$ & \\
\hline Preoperative amiodarone & $5(14 \cdot 3 \%)$ & $6(19 \cdot 4 \%)$ & 0.581 \\
\hline Ischaemic time (min) & 191 (175 to 207$)$ & $177(162$ to 191$)$ & $0 \cdot 193$ \\
\hline Implant time (min) & $77(68$ to 87$)$ & 84 (75 to 93$)$ & 0.352 \\
\hline Bypass time (min) & $127(117$ to 136$)$ & $140(125$ to 154$)$ & $0 \cdot 118$ \\
\hline $\begin{array}{l}\text { Preoperative transpulmonary gradient } \\
(\mathrm{mm} \mathrm{Hg})\end{array}$ & $8 \cdot 2(7 \cdot 1$ to $9 \cdot 2)$ & $7 \cdot 7(5 \cdot 9$ to $9 \cdot 6)$ & 0.671 \\
\hline \multicolumn{3}{|l|}{ Rejection score } & \multirow[t]{4}{*}{0.323} \\
\hline 0 & 11 & 6 & \\
\hline 1 & 14 & 11 & \\
\hline$>1$ & 10 & 14 & \\
\hline
\end{tabular}

Values are number $(\%)$ or mean (95\% confidence interval).

Table 2 Comparison between standard and bicaval groups (arrhythmias and pacing)

\begin{tabular}{|c|c|c|c|}
\hline & Standard & Bicaval & p Value \\
\hline No of patients with temporary pacing & $16(45 \cdot 7)$ & $10(32 \cdot 3)$ & 0.264 \\
\hline No of patients with AT & $13(37 \cdot 1)$ & $3(9 \cdot 7)$ & 0.009 \\
\hline \multicolumn{4}{|l|}{ Type of AT (arrhythmia days) } \\
\hline Atrial fibrillation & 21 & 3 & \\
\hline Atrial flutter & 1 & 1 & \\
\hline Atrial tachycardia & 1 & 0 & \\
\hline SVT & 4 & 0 & \\
\hline $\begin{array}{l}\text { No of patients with treated } \\
\text { atrial tachyarrhythmia }\end{array}$ & $4(11 \cdot 4)$ & $3(9 \cdot 7)$ & $1 \cdot 0$ \\
\hline \multicolumn{4}{|l|}{ Treatment (episodes and indication) } \\
\hline Cardioversion & $\begin{array}{l}2 \text { atrial fibrillation } \\
1 \text { atrial tachycardia, } \\
1 \text { SVT }\end{array}$ & $\begin{array}{l}1 \text { atrial fibrillation } \\
1 \text { atrial tachycardia }\end{array}$ & \\
\hline $\begin{array}{l}\text { Drugs } \\
\text { No of patients with permanent pacing }\end{array}$ & $\begin{array}{l}4 \text { atrial fibrillation } \\
3(8 \cdot 6)\end{array}$ & ${ }_{0}^{2}$ atrial fibrillation & 0.241 \\
\hline $\begin{array}{l}\text { No of days to discharge } \\
\text { (mean ( } 95 \% \text { confidence interval)) }\end{array}$ & $29.1(25 \cdot 7$ to 32.9$)$ & $24 \cdot 1(21 \cdot 6$ to $26 \cdot 8)$ & 0.024 \\
\hline
\end{tabular}

Values in parentheses are percentages.

SVT, supraventricular tachycardia.

AT, atrial tachyarrhythmia. occurred 5 days before or 7 days after obtaining a grade 2 or 3 a rejection biopsy specimen or between two biopsy procedures with specimens showing grade 2 or $3 a$ rejection), whether arrhythmia required treatment, preoperative amiodarone treatment (yes/no), ischaemic time, implantation/cross clamp time, bypass time, and preoperative transpulmonary gradient. A rejection score for each patient was calculated by allocating a score of one for each episode of grade 2 or 3 a rejection and summing the scores for biopsy specimens in the first 30 days. The rejection grade of each biopsy specimen was determined by standard histological techniques and according to internationally agreed criteria. ${ }^{3}$

\section{ORGAN PRESERVATION}

Donor organs were harvested in a standard manner. They were perfused with crystalloid cardioplegia and transported at $4^{\circ} \mathrm{C}$ in normal saline surrounded by crushed ice.

\section{STATISTICAL ANALYSIS}

Patient subgroups were compared initially using the appropriate univariate methods: Fisher's exact test and contingency table analyses for categorical variables and unpaired Student's $t$ test and one way analyses of variance for continuous variables. If the latter had a positively skewed distribution then they were normalised by converting the data to natural logarithms. The relative risks associated with the occurrence of atrial tachyarrhythmias and temporary pacing were estimated by multiple logistic regression analyses. Factors related to days to discharge were investigated using standard multiple regression methods. Results are presented using summary statistics with their $95 \%$ confidence intervals.

\section{DATA COLLECTION AND DEFINITIONS}

Data were retrieved from the case notes, nursing notes, and intensive care charts. These included: sex, age at transplant, primary diagnosis, days to discharge, requirement for temporary pacing (number of days on which pacing occurred-performed using temporary atrial and ventricular epicardial pacing leads implanted routinely at the time of surgery), incidence of atrial tachyarrhythmias (number of days on which atrial tachyarrhythmia occurred), arrhythmia type, days post-transplantation, relation between arrhythmia and rejection (considered positive if arrhythmia

\section{Overall comparison of} bicaval and standard anastomoses for hospital stay, incidence of atrial tachycardia, temporary pacing, permanent pacing, and death. Asterisks indicate statistically significant differences.

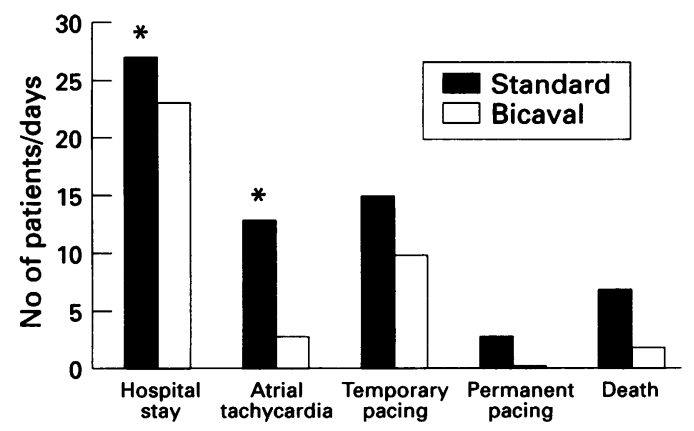

\section{Results}

BICAVAL VERSUS STANDARD ANASTOMOSIS

Between January 1991 and December 1993, 75 heart transplants were performed. Nine patients died during the first 30 days and were excluded from further analysis (none had atrial tachyarrhythmias). The difference between the numbers of deaths in the two groups was not significant $(P=0 \cdot 28)$. Tables 1 and 2 , and the figure, present a comparison between the standard and bicaval anastomoses groups. The groups were well matched in terms of ischaemic times, preoperative transpulmonary gradient, and the preoperative use of amiodarone.

The most pronounced difference was in the incidence of atrial tachyarrhythmias with significantly more occurring in the standard group $(P=0.009)$. There were also more arrhythmias which required treatment (cardioversion or drug treatment) in the standard pacing, although these differences were not statistically significant. Patients in the bicaval group were discharged from hospital significantly earlier on average than those in the standard group $(P=0.024)$. group and more temporary and permanent 
Table 3 Atrial tachyarrhythmias in the first postoperative week

\begin{tabular}{|c|c|c|c|}
\hline & Standard anastomosis & Bicaval anastomosis & $p$ Value \\
\hline $\begin{array}{l}\text { No of patients (\%) } \\
\text { No of episodes (arrhythmia days) } \\
\text { Details of episodes (arrhythmia, } \\
\text { postoperative days, treatment) } \\
\text { (patients are numbered) }\end{array}$ & $\begin{array}{l}10 \text { (29) } \\
\text { 14 } \\
\text { 1. Atrial fibrillation day 3; no treatment } \\
\text { 2. SVT, day } 1 \text {; no treatment } \\
\text { 3. SVT, day } 1 \text {; no treatment } \\
\text { 4. Atrial fibrillation; day 3; no treatment } \\
\text { Atrial fibrillation; day 7; digoxin } \\
\text { 5. Atrial fibrillation; day 5; no treatment } \\
\text { 6. Atrial fibrillation; day 3; intravenous verapamil } \\
\text { 7. Atrial fibrillation; day 3; cardioversion } \\
\text { Atrial tachycardia; day 7; cardioversion } \\
\text { 8. Atrial fibrillation; days 3-5 no treatment } \\
\text { 9. Atrial flutter; day 2; no treatment } \\
\text { 10. SVT; day 1; no treatment }\end{array}$ & $\begin{array}{l}\text { 1 (3) } \\
1 \text { Atrial fibrillation; day } 3 \text {; } \\
\text { intravenous verapamil }\end{array}$ & 0.006 \\
\hline
\end{tabular}

SVT, supraventricular tachycardia.

Table 4 Relative risk of atrial tachyarrhythmia due to different factors

\begin{tabular}{lll}
\hline Factor & Relative risk & $p$ Value \\
\hline Anastomosis (standard relative to bicaval) & $5.52(1.36$ to 22.4$)$ & 0.015 \\
Sex (male relative to female) & $3.29(0.37$ to 29.5$)$ & 0.277 \\
Age (increase of 1 year) & $1.02(0.97$ to 1.08$)$ & 0.361 \\
Diagnosis (IHD relative to IDC) & $1.47(0.43$ to 5.06$)$ & 0.536 \\
Amiodarone & $0.27(0.03$ to 2.37$)$ & 0.226 \\
Ischaemic time (increase of 30 min) & $1.06(0.74$ to 1.52$)$ & 0.751 \\
Implant time (increase of $30 \mathrm{~min})$ & $1.10(0.70$ to 1.71$)$ & 0.677 \\
Bypass time (increase of $30 \mathrm{~min})$ & $0.94(0.71$ to 1.26$)$ & 0.686 \\
Preoperative TPG (increase of $5 \mathrm{~mm} \mathrm{Hg})$ & $1.28(0.66$ to 2.48$)$ & 0.464 \\
\hline
\end{tabular}

Values are relative risk ( $95 \%$ confidence interval); IHD, ischaemic heart disease; IDC, idiopathic dilated cardiomyopathy; TPG, transpulmonary gradient.

Table 5 Relative risk of temporary pacing due to different factors

\begin{tabular}{lll}
\hline Factor & Relative risk & $p$ Value \\
\hline Anastomosis (standard relative to bicaval) & $1.77(0.63$ to 4.93$)$ & 0.266 \\
Sex (male relative to female) & $0.97(0.24$ to 3.94$)$ & 0.966 \\
Age (increase of 1 year) & $0.98(0.95$ to 1.02$)$ & 0.410 \\
Diagnosis (IHD relative to IDC) & $0.71(0.24$ to 2.07$)$ & 0.523 \\
Amiodarone & $3.32(0.84$ to 13.1$)$ & 0.082 \\
Ischaemic time (increase of $30 \mathrm{~min})$ & $1.08(0.78$ to 1.48$)$ & 0.645 \\
Implant time (increase of $30 \mathrm{~min})$ & $1.10(0.75$ to 1.63$)$ & 0.608 \\
Bypass time (increase of $30 \mathrm{~min})$ & $1.14(0.87$ to 1.49$)$ & 0.341 \\
Preoperative TPG (increase of $5 \mathrm{~mm} \mathrm{Hg})$ & $0.79(0.40$ to 1.57$)$ & 0.490
\end{tabular}

Values are relative risk ( $95 \%$ confidence interval); IHD, ischaemic heart disease; IDC, idiopathic dilated cardiomyopathy; TPG, transpulmonary gradient.

Table 6 Influence of different factors on days to discharge

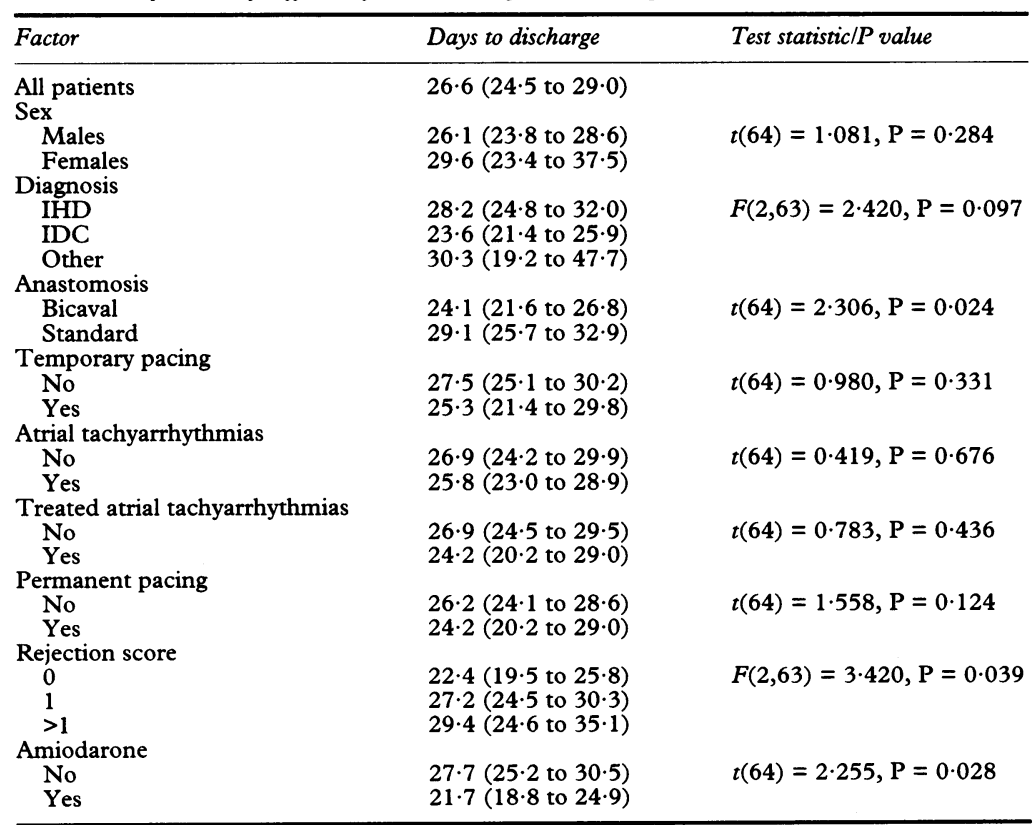

Values are mean ( $95 \%$ confidence interval); IHD = ischaemic heart disease; IDC = idiopathic dilated cardiomyopathy.
Table 3 presents a further analysis comparing atrial tachyarrhythmias between the two groups for the first 7 days post-transplantation. This period was subjected to separate analysis as continuous telemetric monitoring was available for all patients during this period; it is possible that some asymptomatic arrhythmias might have been missed in the later period. The results were similar to the overall analysis; 14 episodes of atrial tachyarrhythmia occurred in 10 patients in the standard group compared with one episode in the bicaval group $(P=0.006)$.

\section{ATRIAL TACHYARRHYTHMIAS}

Table 4 gives the results of the relative risk analysis for the incidence of atrial tachyarrhythmias. The only factor that significantly affected the incidence of atrial tachyarrhythmias was standard atrial anastomosis; this was associated with more than five times the risk of atrial tachyarrhythmia.

Most episodes of atrial tachyarrhythmia occurred within the first postoperative week. Those who experienced arrhythmias did not have higher rejection scores than those who did (arrhythmia median (range) rejection score 1 (0-2); no arrhythmia median (range) rejection score $1(0-3) ; P=0 \cdot 26)$, although in seven episodes in three patients (six episodes of atrial fibrillation and one of atrial flutter), the arrhythmia seemed temporally related to rejection.

\section{TEMPORARY PACING}

Table 5 gives the results of the relative risk analysis for temporary pacing. No preoperative or operative factor caused a statistically significant increase in the risk of temporary pacing, although preoperative use of amiodarone approached significance (relative risk $3.32, P=0.082$ ). Standard anastomosis produced an increased relative risk of temporary pacing (1.77), but this was not significant $(P=0 \cdot 266)$.

\section{DAYS TO DISCHARGE}

Table 6 gives the results of the univariate analysis of days to discharge. Three factors were significant: bicaval compared with standard anastomosis, rejection score, and preoperative use of amiodarone. Multivariate analysis showed that type of anastomosis and rejection score were independently associated 
with days to discharge; standard anastomosis and incidence of rejection were associated with longer hospital stays.

PERMANENT PACING

Three permanent pacing systems were implanted: two ventricular and one dual chamber, (all rate adaptive). Two were implanted for symptomatic sinus bradycardia (fatigue and shortness of breath) at 38 days and 160 days, and one at 712 days for complete heart block and syncope.

\section{VENTRICULAR ARRHYTHMIAS}

Ventricular arrhythmias were also observed. In the standard group there were seven episodes in five patients (four non-sustained ventricular tachycardia, one sustained ventricular tachycardia, and two episodes of ventricular fibrillation). There were two episodes of non-sustained ventricular tachycardia in two patients in the bicaval group.

\section{Discussion}

Several studies have examined the incidence of atrial arrhythmias and pacing after heart transplantation. ${ }^{4-10}$

Romhilt et $a l^{4}$ studied 13 long-term survivors of cardiac transplantation. Three (23\%) patients experienced non-sustained paroxysmal supraventricular tachycardia within the first 30 days, five (38\%) experienced sinus bradycardia (undefined), and three $(23 \%)$ subsequently required implantation of a permanent pacing system. Monitoring was by daily electrocardiograms. No atrial fibrillation, flutter, or tachycardia was documented.

Scott $e t a l^{5}$ studied 50 patients surviving for more than 2 weeks after transplantation by continuous telemetry while in hospital. Nine (18\%) had sustained atrial tachyarrhythmias (atrial fibrillation or atrial flutter) in the first 6 weeks; non-sustained atrial tachyarrhythmias occurred in nine patients and non-sustained supraventricular tachycardia in nine, although it is not clear whether these were the same nine patients in whom sustained arrhythmias were observed. In this study sustained atrial flutter was significantly associated with rejection. Bradyarrhythmias and pacing were not discussed.

Jacquet et $a l^{6}$ used telemetry to study 25 transplant patients (including three who died early); mean follow up was 28 days. Supraventricular arrhythmias (atrial fibrillation, atrial flutter, sustained and non-sustained supraventricular tachycardia) occurred in 11 patients $(44 \%)$. Ten $(40 \%)$ patients required temporary pacing. Two patients $(8 \%)$ required permanent pacing for sinus node dysfunction. No relation between atrial arrhythmias and rejection was demonstrated.

These studies indicate an incidence of atrial tachyarrhythmias soon after orthotopic heart transplantation of between 18 and $44 \%$. Jacquet $e t a l^{6}$ reported an incidence of $44 \%$, using definitions and monitoring similar to those of the present study. We observed an overall incidence of $24 \%$, with $38 \%$ among patients having standard anastomoses and $10 \%$ among the bicaval group. The incidence of AT in our standard anastomosis group is comparable with that of Jacquet et al. ${ }^{6}$

We sought an explanation for the incidence of atrial tachyarrhythmias in terms of proximity to the operation and relation to rejection. Arrhythmia occurred within 1 week after operation in most patients $(69 \%)$ with abnormal rhythm. Arrhythmia was temporally related to rejection in only three of 16 patients $(19 \%)$, in two of whom arrhythmia occurred after the first week. Seven patients required treatment for atrial tachyarrhythmia, which occurred in the first week in four and was related to rejection in one (although this patient experienced two episodes, each requiring treatment). Rejection scores of those patients who had arrhythmias were not significantly different from those who did not. This does not support a general association of atrial tachyarrhythmias with rejection, though previous reports ${ }^{5}$ have emphasised that sustained atrial flutter is the arrhythmia particularly associated with rejection. We observed only two episodes of atrial flutter, one of which required treatment and was related to rejection.

Previously described rates of $38 \%$ for early bradycardias $^{4}$ or $40 \%$ for temporary pacing 6 are similar to $39 \%$ that we observed for temporary postoperative pacing (bicaval 32\%, standard $46 \%$ ), but bradycardias were not separately documented as we considered that this would have been unreliable in a retrospective study. Early pacing was well documented, and any significant bradycardia is likely to have been treated by pacing.

Reports of the need for permanent pacing after heart transplantation vary from $8 \%$ to $21 \%$ of patients. ${ }^{6-9}$ These differences may in part be explained by initial concerns that, after transplantation, patients with sinoatrial disease have a poor prognosis and that early pacing should be undertaken. ${ }^{10}$ This has not been supported by subsequent experience and our policy has been to avoid implanting permanent systems, if possible, until at least 3 weeks have elapsed. Our overall rate of permanent pacing was $5 \%$ but all three of these pacing systems were implanted among the standard anastomosis group giving a rate of $9 \%$ in this group, comparable with $11 \%$ cited in two recent studies. ${ }^{89}$

We identified anastomosis type and rejection score as significantly and independently associated with length of hospital stay; patients in the bicaval group had a shorter hospital stay as did patients with fewer rejection episodes. The finding that patients with more rejection episodes had a longer hospital stay accords with general experience, as such patients are detained until satisfactory control of rejection is achieved (regardless of their clinical condition in other respects). The shorter hospital stay of those receiving bicaval anastomosis suggests that the superior atrial configuration (right and left) resulting from this technique is important. The lower incidence 
of tachyarrhythmias which we have demonstrated may contribute to the superiority of this procedure, but it is more likely to be related to superior ventricular filling and a reduced tendency to mitral and tricuspid regurgitation which might be expected with this technique and which has been described with the combination of bicaval and pulmonary vein anastomoses. ${ }^{11} 12$ This probably confers a small but important advantage for patients who are delicately poised between recovery and complications. While we are cautious in accepting this finding it has potential importance for patients and hospital costs associated with the operation. We note that other studies on variants of anastomotic technique have not assessed discharge time ${ }^{11} 12$ and we suggest that this may be a useful early marker for a clinical benefit which may otherwise be difficult to define using relatively small numbers of patients.

In considering other variables which might have influenced the occurrence of bradycardias or tachyarrhythmias, we examined the influence of preoperative use of amiodarone. A previous study has reported an increase in postoperative pacing requirement after transplantation when the recipient received preoperative amiodarone. ${ }^{13} \mathrm{We}$ also found that a higher proportion of those who received amiodarone required pacing than those who did not and that preoperative amiodarone conferred an estimated relative risk of temporary pacing of 3.32 compared with those not given amiodarone; this was not significant but there was a highly suggestive trend $(P=0.082)$. Patients who received amiodarone also had a lower relative risk of developing atrial tachyarrhythmias, although this was not statistically significant (table 4).

Other groups have described modification of the standard technique of transplantation with the use of "atrioventricular"11 or "total orthotopic" transplantation ${ }^{12} 14$; the technique in each case consists of bicaval anastomosis combined with separate anastomosis of the pulmonary veins. In the study of Kendall $e t a l^{11}$ the technique was found to reduce tricuspid incompetence but no functional improvement in patient status was shown and the technique was considered more technically demanding without important advantages; the occurrence of arrhythmias was not reported nor was the length of hospital stay. Czer et al ${ }^{12}$ reported elimination of symptomatic bradyarrhythmias requiring the use of a permanent pacemaker, as well as a reduction in mitral and tricuspid regurgitation. The principal disadvantage of these techniques compared with that we have described is that there is little access to the medial aspect of the pulmonary vein anastomoses in the event of postoperative bleeding. In a recent study of three cases Czer's group ${ }^{15}$ have also recently described a bicaval technique identical to that which we have described here (and previously reported elsewhere $^{216}$ ) and commend its simplicity and potential advantages.

This study has a number of limitations. The allocation of technique was not formally randomised; data were collected retrospectively; continuous monitoring was not employed throughout the period (although telemetry was used for all patients during their intensive care and early postoperative period, together with accurate and detailed recording of cardiac rhythm and pacing, as well as recording of events of clinical importance). Despite these concerns examination of the two groups for factors likely to affect the variables of interest reveals that they were well matched. It is likely that valid conclusions can be drawn about differences in the events studied between the two anastomotic techniques.

In summary we have shown a significant decrease in the incidence of atrial tachyarrhythmias with the technique of bicaval anastomosis and a significant decrease in days to discharge. Moreover, the need for permanent and temporary pacing and treatment of atrial tachyarrhythmias were also less in the bicaval group, although they were not statistically significant. These observations support the hypothesis that the sinus node is at less risk from this surgical approach and that a less arrhythmogenic atrial substrate is created.

We thank all the surgeons involved with the transplant programme and in particular $\mathrm{Mr} \mathrm{C} \mathrm{S} \mathrm{Campbell,} \mathrm{Mr} \mathrm{A} \mathrm{K}$ Deiraniya, and Mr A N Rahman.

1 Lower RR, Stofer RC, Shumway NE. Homovital transplantation of the heart. $\mathcal{F}$ Thorac Cardiovasc Surg 1961 41:196-202.

2 Sarsam MAI, Campbell CS, Yonan NA, Deiraniya AK, Rahman AN. An alternative surgical technique in orthotopic cardiac transplantation. $\mathcal{F}$ Cardiovasc Surg 1993;8 344-9.

3 Billingham ME, Cary NRB, Hammond ME, et al. A working formulation for the standardisation of nomenclature in the diagnosis of heart and lung rejection: Heart Rejection Study Group. I Heart Lung Transpl 1990;9: 587-93.

4 Romhilt DW, Doyle M, Sagar KB, et al. Prevalence and significance of arrhythmias in long-term survivors of cardiac transplantation. Circulation 1982;66:I219-2.

5 Scott CD, Dark JH, McComb J. Arrhythmias after cardiac transplantation. Am f Cardiol 1992;70:1061-3.

6 Jacquet L, Ziady G, Stein K, et al. Cardiac rhythm disturbances early after orthotopic heart transplantation prevalence and clinical importance of the observed abnormalities. F Am Coll Cardiol 1990;16:832-7.

7 Heinz G, Hirschl M, Buxbaum P, Laufer G, Gasic S, Laczkovics A. Sinus node dysfunction after orthotopic cardiac transplantation: postoperative incidence and cardiac transplantation: postoperative inciden
long term implications. $P A C E$ 1992;15:731-7.

8 Markewitz A, Schmoeckel M, Nollert G, Uberfuhr P, Weinhold C, Reichart B. Long term results of pacemaker therapy after orthotopic heart transplantation. $f$ Card Surg 1993;8:411-6.

9 Scott CD, McComb J, Dark JH, Bexton RS. Permanen pacing after cardiac transplantation. Br Heart $\mathcal{F} 1993 ; 69$ : 399-403.

10 Mackintosh AF, Carmichael DJ, Wren C, Cory-Pearce R, English TAH. Sinus node function in the first three weeks after cardiac transplantation. Br Heart $\mathcal{F} 1982 ; 48$ : 584-8.

11 Kendall SW, Ciulli F, Biocina B, et al. Atrioventricular orthotopic heart transplantation: a prospective randomised clinical trial in 60 consecutive patients. Transplant Proc 1993;25:1172-3.

12 Czer LSC, Trento A, Blanche C. Orthotopic heart transplantation: clinical experience with a new technique [abstract]. $\mathcal{F ~ A m}$ Coll Cardiol 1993;21 (suppl A):168.

13 Chelimsky-Fallick C, Middlekauff HR, Stevenson WG, $e$ al. Amiodarone therapy does not compromise subsequent heart transplantation. $\mathcal{F} \mathrm{Am}$ Coll Cardiol 1992;20: 1556-61.

14 Dreyfus G, Jebara V, Mihailenau S, Carpentier AF. Total orthotopic heart transplantation: an alternative to the standard technique. Ann Thorac Surg 1991;52:1181-4.

15 Blanche C, Czer LSC, Valenza M, Trento A. Alternative technique for orthotopic heart transplantation. Ann Thorac Surg 1994;57:765-7.

16 Grant SCD, Khan MA, Yonan N, Brooks NH. Technique of anastomosis and incidence of atrial tachyarrhythmias following heart transplantation. $\mathrm{Br}$ Heart $\mathcal{f} 1994$ 71(suppl):40. 